\title{
Two novel classes of solvable many-body problems of goldfish type with constraints
}

\author{
Francesco Calogero* \\ Dipartimento di Fisica, Università di Roma "La Sapienza", 00185 Rome, Italy \\ Istituto Nazionale di Fisica Nucleare, Sezione di Roma. \\ David Gómez-Ullate ${ }^{\dagger}$ \\ Departamento de Física Teórica II \\ Universidad Complutense \\ 28040 Madrid, Spain. \\ January 16,2007
}

\begin{abstract}
Two novel classes of many-body models with nonlinear interactions "of goldfish type" are introduced. They are solvable provided the initial data satisfy a single constraint (in one case; in the other, two constraints): i. e., for such initial data the solution of their initialvalue problem can be achieved via algebraic operations, such as finding the eigenvalues of given matrices or equivalently the zeros of known polynomials. Entirely isochronous versions of some of these models are also exhibited: i.e., versions of these models whose nonsingular solutions are all completely periodic with the same period.
\end{abstract}

\footnotetext{
*francesco.calogero@roma1.infn.it, francesco.calogero@uniroma1.it

$\dagger$ david.gomez-ullate@fis.ucm.es
} 


\section{Introduction and main results}

Long time ago the possibility was noticed of using the nonlinear mapping among the zeros and the coefficients of a polynomial in order to identify solvable (classes of) many-body problems, characterized by nonlinear equations of motions of Newtonian type (with one-body and two-body, generally velocity-dependent, forces) [1,2]. [Terminology: here and hereafter we denote as solvable any problem whose solution can be achieved by algebraic operations, such as finding the zeros of polynomials]. The starting point of these developments is a linear PDE such as

$$
\begin{array}{r}
\psi_{t t}-\left[a_{1}-(N-1) a_{6} z\right] \psi_{t}+\left[a_{2}+a_{3} z-2(N-1) a_{10} z^{2}\right] \psi_{z} \\
-\left(a_{4}+a_{5} z+a_{6} z^{2}\right) \psi_{z t}+\left(a_{7}+a_{8} z+a_{9} z^{2}+a_{10} z^{3}\right) \psi_{z z} \\
-N\left[a_{3}+(N-1)\left(a_{9}-a_{10} z\right)\right] \psi=0 .
\end{array}
$$

[Notation: hereafter $N$ is an arbitrary positive integer $(N \geq 2)$, the symbols $a_{j}$ and (see below) $A_{j}, B_{j}$ denote (a priori arbitrary) constants, and subscripted variables denote partial differentiations of the dependent variable $\psi(z, t)$; note incidentally that this PDE coincides, up to trivial notational changes, with eq. (2.3.3-1) of Ref. [2]]. One then notes that this PDE admits solutions which are (for all time) a monic polynomial of degree $N$ in $z$, and introduces the $N$ zeros $z_{n}(t)$ and the $N$ coefficients $c_{m}(t)$ of such a polynomial solution,

$$
\psi(z, t)=\prod_{n=1}^{N}\left[z-z_{n}(t)\right]=z^{N}+\sum_{m=1}^{N} c_{m}(t) z^{N-m} .
$$

It is then easily seen that the fact that $\psi(z, t)$ satisfies the linear PDE (1) implies that the coefficients $c_{m}(t)$ evolve according to the following system of linear ODEs [2]:

$$
\begin{aligned}
& \ddot{c}_{m}-(N+1-m) a_{4} \dot{c}_{m-1}-\left[(N-m) a_{5}+a_{1}\right] \dot{c}_{m}+m a_{6} \dot{c}_{m+1} \\
& +(N+1-m)(N+2-m) a_{7} c_{m-2}+(N+1-m)\left[(N-m) a_{8}+a_{2}\right] c_{m-1} \\
& -m\left[(2 N-1-m) a_{9}+a_{3}\right] c_{m}+m(m+1) a_{10} c_{m+1}=0 .
\end{aligned}
$$


Likewise, it can be shown (see Ref. [2], or Section 2 below) that the zeros $z_{n}(t)$ evolve according to the following system of nonlinear PDEs

$$
\begin{aligned}
\ddot{z}_{n}= & a_{1} \dot{z}_{n}+a_{2}+a_{3} z_{n}-2(N-1) a_{10} z_{n}^{2} \\
& +\sum_{\substack{m=1 \\
m \neq n}}^{N}\left\{( z _ { n } - z _ { m } ) ^ { - 1 } \left[2 \dot{z}_{n} \dot{z}_{m}+\left(\dot{z}_{n}+\dot{z}_{m}\right)\left(a_{4}+a_{5} z_{n}\right)\right.\right. \\
& \left.\left.+a_{6}\left(\dot{z}_{n} z_{m}+\dot{z}_{m} z_{n}\right) z_{n}+2\left(a_{7}+a_{8} z_{n}+a_{9} z_{n}^{2}+a_{10} z_{n}^{3}\right)\right]\right\} .
\end{aligned}
$$

Notation and terminology: hereafter indices such as $m, n$ range from 1 to $N$ (unless otherwise indicated), superimposed dots denote differentiations with respect to the time $t$, and the $N$ zeros $z_{n}(t)$ are interpreted as the coordinates of $N$ "point particles" evolving according to the "Newtonian equations of motion" (4) featuring one-body and two-body velocity dependent forces; and an analogous interpretation shall be given in all analogous cases below. Unless otherwise explicitly specified, we hereafter assume that all quantities under consideration (except the time $t$ ) are generally complex numbers, so that the motions of the particles characterized by the coordinates $z_{n}(t)$ take place in the complex $z$-plane; of course such motions can easily be reinterpreted as taking place in a "more physical" real plane, but we shall not devote space to discuss this well-known aspect (see, for instance, Chapter 4 of Ref. [2], entitled "Solvable and or integrable many-body problems in the plane, obtained by complexification").

Clearly, since the parameters $a_{j}$ are (arbitrary) constants, the solution of the system of linear ODEs (3) satisfied by the coefficients $c_{m}(t)$ can be achieved by purely algebraic operations; and once the $N$ coefficients $c_{m}(t)$ of the polynomial $\psi(z, t)$ have been obtained, the computation of the $N$ zeros $z_{n}(t)$ of this polynomial, see (2), is also a purely algebraic task. Hence the determination of the $N$ coordinates $z_{n}(t)$ whose time evolution is characterized by the Newtonian equations of motion (44) can be achieved via purely algebraic operations: this $N$-body problem is solvable.

The 10-parameter class of solvable $N$-body models (4) is vast, and it includes several interesting cases [2]. Even the very simplest case, characterized by the vanishing of all the parameters $a_{j}$, is quite remarkable: in this case the Newtonian equations of motion read simply

$$
\ddot{z}_{n}=\sum_{\substack{m=1 \\ m \neq n}}^{N} \frac{2 \dot{z}_{n} \dot{z}_{m}}{z_{n}-z_{m}}
$$


and the solution of the initial-value problem for this problem is given by the following simple proposition: the $N$ coordinates $z_{n}(t)$ are the $N$ zeros of the following algebraic equation in $z$,

$$
\sum_{n=1}^{N} \frac{\dot{z}_{n}(0)}{z-z_{n}(0)}=\frac{1}{t}
$$

Note that, by multiplying this equation by the product $\prod_{n=1}^{N}\left[z-z_{n}(0)\right]$, it becomes a polynomial equation, with time-dependent coefficients, of degree $N$ in $z$, which indeed generally has $N$ solutions, at least if one allows the coordinates $z_{n}$ to be complex numbers, hence their time-evolution to take place in the complex z-plane; indeed such an evolution can also be reinterpreted as the motion of $N$ point particles on a real ("physical") plane, and its phenomenology is quite amusing, see Section 2.4.2 of Ref. [2], entitled "The simplest model: explicit solution (the game of musical chairs), Hamiltonian structure". Because of these remarkable features, as well as the neat form of the Newtonian equations of motion (5a) (which also happen to be Hamiltonian), this $N$-body model was given the honorary title of "goldfish" [3], and this attribute was then extended to several solvable models featuring in the right-hand side of their Newtonian equations of motion ("acceleration equal force") terms such as that appearing in the right-hand side of (5a): note that these solvable models "of goldfish type" include now the models belonging to the 10-parameter class (4) and several models outside of this class (for an updated review of such models see [4]).

In the present paper we exhibit two classes of solvable $N$-body models "of goldfish type" which are, to the best of our knowledge, new, hence, in particular, not included in the class (44). These novel models are however solvable only provided the particle coordinates $z_{n}(t)$ satisfy additional conditions, which must of course be compatible with the time evolution, so that it is sufficient that they be satisfied, in the context of the initial-value problem, by the initial data. This limitation could be considered as a drawback of these new models, or instead as a feature that adds to their interest in the context of mathematical physics. The hunch that such models should exist was suggested to us by recent results [7-11] concerning certain exceptional polynomial subspaces, as tersely reviewed in Appendix A.

The first class of these models is characterized by the following (6-parameter) 
Newtonian equations of motion:

$$
\begin{aligned}
\ddot{z}_{n}= & A_{1} \dot{z}_{n}+A_{2}+A_{3} z_{n}-\frac{2 A_{4}}{z_{n}} \\
& +\sum_{\substack{m=1 \\
m \neq n}}^{N}\left\{( z _ { n } - z _ { m } ) ^ { - 1 } \left[2 \dot{z}_{n} \dot{z}_{m}+A_{5}\left(\dot{z}_{n}+\dot{z}_{m}\right) z_{n}\right.\right. \\
& \left.\left.+2\left(A_{4}-A_{2} z_{n}+A_{6} z_{n}^{2}\right)\right]\right\} .
\end{aligned}
$$

Here the novel element - not included in the class (44) - is that associated with the constant $A_{4}$. In the following section we show that these equations of motion entail that the coefficients $c_{m}(t)$ - related to the coordinates $z_{n}(t)$ via the mapping (2) - evolve according to the following system of linear ODEs:

$$
\begin{aligned}
\ddot{c}_{m} & -\left[(N-m) A_{5}+A_{1}\right] \dot{c}_{m}+(N-1-m)(N+2-m) A_{4} c_{m-2} \\
& -(N+1-m)(N-1-m) A_{2} c_{m-1} \\
& -m\left[(2 N-1-m) A_{6}+A_{3}\right] c_{m}=0
\end{aligned}
$$

of course with $c_{0}=1$ and $c_{m}=0$ for $m>N$ and $m<0$ (consistently with (2), and also with this system of ODEs). Note that the linear character of this system of ODEs entails the solvable character of the Newtonian equations of motion (6). But these findings are only valid if the coordinates $z_{n}(t)$ satisfy the constraint

$$
\sum_{n=1}^{N} \frac{1}{z_{n}(t)}=0
$$

and correspondingly (see below) the coefficients $c_{m}(t)$ satisfy the constraint

$$
c_{N-1}(t)=0 .
$$

The treatment of Section 2 implies that these constraints, (8) respectively (9), are indeed compatible with the evolution equations (6) respectively (7); hence they are automatically satisfied, in the context of the initial-value problem, provided the initial data for the $N$-body problem (66) satisfy the following two conditions:

$$
\sum_{n=1}^{N} \frac{1}{z_{n}(0)}=0, \quad \sum_{n=1}^{N} \frac{\dot{z}_{n}(0)}{\left[z_{n}(0)\right]^{2}}=0
$$


and correspondingly the initial data for the linear system of ODEs (7) satisfy the two conditions

$$
c_{N-1}(0)=\dot{c}_{N-1}(0)=0 .
$$

Before turning to the second class of new models, let us display an equivalent avatar of the Newtonian equations of motion (6) :

$$
\begin{aligned}
\ddot{z}_{n} & =\left(A_{1}+\frac{N-2}{2} A_{5}\right) \dot{z}_{n}-(N-2) A_{2}+\left[A_{3}+(N-2) A_{6}\right] z_{n}-\frac{2 A_{4}}{z_{n}} \\
& +\frac{A_{5}}{2} \dot{Z}+A_{6} Z+\sum_{\substack{m=1 \\
m \neq n}}^{N}\left\{( z _ { n } - z _ { m } ) ^ { - 1 } \left[2 \dot{z}_{n} \dot{z}_{m}+\frac{A_{5}}{2}\left(\dot{z}_{n}+\dot{z}_{m}\right)\left(z_{n}+z_{m}\right)\right.\right. \\
& \left.\left.+2 A_{4}-A_{2}\left(z_{n}+z_{m}\right)+A_{6}\left(z_{n}^{2}+z_{m}^{2}\right)\right]\right\},
\end{aligned}
$$

where

$$
Z(t)=\sum_{n=1}^{N} z_{n}(t) .
$$

[This second version, (12) with (13), has the merit to immediately yield (by summing over $n$ from 1 to $N$ - using (8) and the vanishing of the double sum in the right-hand side due to the antisymmetry of the summand under the exchange of the dummy indices $m$ and $n$ ) the linear ODE

$$
\ddot{Z}=\left[A_{1}+(N-1) A_{5}\right] \dot{Z}-N(N-2) A_{2}+\left[A_{3}+2(N-1) A_{6}\right] Z ;
$$

which is consistent, via the relation

$$
c_{1}(t)=-Z(t)
$$

implied by (2), with the equation satisfied by $c_{1}(t)$ that obtains by setting $m=1$ in (77). On the other hand the first version, (66), has the merit to feature only one-body and two-body forces].

The second class of new models is characterized by the following (7-parameter) Newtonian equations of motion:

$$
\begin{aligned}
\ddot{z}_{n}= & B_{1} \dot{z}_{n}-(N-1) B_{2} z_{n}-2(N-1) B_{3} z_{n}^{2}+B_{4} \frac{\dot{z}_{n}}{z_{n}} \\
& +\sum_{\substack{m=1 \\
m \neq n}}^{N}\left\{( z _ { n } - z _ { m } ) ^ { - 1 } \left[2 \dot{z}_{n} \dot{z}_{m}+\left(\dot{z}_{n}+\dot{z}_{m}\right)\left(B_{4}+B_{5} z_{n}\right)\right.\right. \\
& \left.\left.+B_{6}\left(\dot{z}_{n} z_{m}+\dot{z}_{m} z_{n}\right) z_{n}+2\left(B_{7} z_{n}+B_{2} z_{n}^{2}+B_{3} z_{n}^{3}\right)\right]\right\} .
\end{aligned}
$$


Clearly the novel element (in (15) relative to (4)) is now that associated with the constant $B_{4}$. In the following section we show that these equations of motion, (15), entail that the coefficients $c_{m}(t)$ - related to the coordinates $z_{n}(t)$ via the mapping (2) - evolve as follows:

$$
\begin{array}{r}
\ddot{c}_{m}-(N-m) B_{4} \dot{c}_{m-1}-\left[(N-m) B_{5}+B_{1}\right] \dot{c}_{m}+m B_{6} \dot{c}_{m+1} \\
+(N+1-m)(N-m) B_{7} c_{m-1} \\
-m(N-m) B_{2} c_{m}+m(m+1) B_{3} c_{m+1}=0,
\end{array}
$$

entailing the solvable character of the Newtonian equations of motion (6). But this conclusion is valid only if the coordinates $z_{n}(t)$ satisfy the constraint

$$
\sum_{n=1}^{N} \frac{\dot{z}_{n}(t)}{z_{n}(t)}=0,
$$

and correspondingly (see below) the coefficients $c_{m}(t)$ satisfy the constraint

$$
\dot{c}_{N}(t)=0 .
$$

The treatment of Section 2 implies that these constraints are indeed compatible with the evolution equations (15) and (16); hence they are automatically satisfied, in the context of the initial-value problem, provided the initial data for the $N$-body problem (15) satisfy the following single condition:

$$
\sum_{n=1}^{N} \frac{\dot{z}_{n}(0)}{z_{n}(0)}=0,
$$

and correspondingly the initial data for the linear system of ODEs (16) satisfy the single condition

$$
\dot{c}_{N}(0)=0 \text {. }
$$

The paper is organized as follows. In Section 2 the results reported above are proven. In Sections 3 and 4 we discuss tersely the behavior of these two solvable $N$-body problems, and in Section 5 entirely isochronous versions of these novel models are identified: they are characterized by the fact that all their nonsingular solutions are completely periodic (namely, periodic in all their dependent variables $z_{n}(t)$ ) with the same basic period (or possibly with a - generally not too large [12] - integer multiple of it). The last Section 6 outlines tersely further developments, to be reported in future publications. 
The paper is completed by two Appendices: in the first one certain relevant results concerning exceptional polynomial subspaces are tersely reviewed, in the second one certain computations are confined whose treatment in the body of the paper would disrupt the flow of the presentation.

\section{Proofs}

The procedure to arrive at the results reported above is by now textbook material $[2,4]$, hence our treatment here can be terse; although the new twist should be emphasized, implying that the solvable character of the new models applies only provided their time evolution is somewhat restricted - as already indicated in the preceding section. The starting point of our treatment is the linear PDE (but see the Remark below)

$$
\begin{aligned}
\psi_{t t} & -\left[a_{1}-(N-1) z a_{6}\right] \psi_{t}+\left[a_{2}+a_{3} z-2(N-1) a_{10}\right] \psi_{z} \\
- & \left(a_{4}+a_{5} z+a_{6} z^{2}\right) \psi_{z t}+\left(a_{7}+a_{8} z+a_{9} z^{2}+a_{10} z^{3}\right) \psi_{z z} \\
- & N\left[a_{3}+(N-1)\left(a_{9}-a_{10} z\right)\right] \psi \\
& =\frac{a_{11}}{z}\left[\psi_{t}-\frac{\dot{c}_{N}}{c_{N}}\right]+\frac{a_{12}}{z}\left[\psi_{z}-\frac{c_{N-1}}{c_{N}}\right] .
\end{aligned}
$$

[Notation: the symbols $a_{j}$ denote again (a priori arbitrary) constants, subscripted variables denote partial differentiations, and the dependent variable $\psi(z, t)$, as well as the coefficients $c_{N}(t)$ and $c_{N-1}(t)$, are characterized by (2)].

Remark. The consistency of this evolution PDE, (21), with the fact that the dependent variable $\psi(z, t)$ is the monic polynomial (2) of degree $N$ in $z$ shall be clear from what follows. Note however that this evolution equation, (21), is a linear PDE iff the two constants $a_{11}$ and $a_{12}$ vanish (in which case it coincides with (1D)); otherwise it is in fact a nonlinear functional equation, as implied by the formulas (clearly entailed by (2))

$$
c_{N}(t)=\psi(0, t), \quad c_{N-1}(t)=\psi_{z}(0, t) .
$$

Via (2), this evolution equation (21) entails 1 the following (systems of)

\footnotetext{
${ }^{1}$ The derivation of the first of these two systems of ODEs is a rather trivial exercise; the derivation of the second system is as well trivial, but readers who find this second task exceedingly painstaking are advised to use the formulas given in Section 2.3.2 of Ref. [2], or (even more conveniently) in Appendix A of Ref. [4].
} 
evolution ODEs for the $N$ coefficients $c_{m}(t)$ and for the $N$ zeros $z_{n}(t)$ :

$$
\begin{array}{r}
\ddot{c}_{m}-(N+1-m) a_{4} \dot{c}_{m-1}-\left[(N-m) a_{5}+a_{1}\right] \dot{c}_{m}+m a_{6} \dot{c}_{m+1} \\
+(N+1-m)(N+2-m) a_{7} c_{m-2}+(N+1-m)\left[(N-m) a_{8}+a_{2}\right] c_{m-1} \\
-m\left[(2 N-1-m) a_{9}+a_{3}\right] c_{m}+m(m+1) a_{10} c_{m+1} \\
=a_{11}\left(\dot{c}_{m-1}-\frac{\dot{c}_{N}}{c_{N}} c_{m-1}\right)+a_{12}\left[(N+2-m) c_{m-2}-\frac{c_{N-1}}{c_{N}} c_{m-1}\right],(23) \\
\ddot{z}_{n}=a_{1} \dot{z}_{n}+a_{2}+a_{3} z_{n}-2(N-1) a_{10} z_{n}^{2}+a_{11} \frac{\dot{z}_{n}}{z_{n}}-\frac{a_{12}}{z_{n}} \\
\quad+\sum_{\substack{m=1 \\
m \neq n}}^{N}\left\{( z _ { n } - z _ { m } ) ^ { - 1 } \left[2 \dot{z}_{n} \dot{z}_{m}+\left(\dot{z}_{n}+\dot{z}_{m}\right)\left(a_{4}+a_{5} z_{n}\right)\right.\right. \\
\left.\left.+a_{6}\left(\dot{z}_{n} z_{m}+\dot{z}_{m} z_{n}\right) z_{n}+2\left(a_{7}+a_{8} z_{n}+a_{9} z_{n}^{2}+a_{10} z_{n}^{3}\right)\right]\right\} .(24)
\end{array}
$$

The observation that the system (23) is consistent with $c_{0}=1$ and $c_{m}=0$ for $m>N$ and $m<0$ confirms the consistency with the evolution equation (21) of the assumption that $\psi(z, t)$ be a monic polynomial of degree $N$ in $z$, see (2).

In order to guarantee that these Newtonian equations of motion, (24), be solvable, we must make sure that the corresponding evolution equations (23) be effectively linear. A straightforward way to achieve this goal is to set to zero the two parameters $a_{11}$ and $a_{12}$; but this simply reproduces the previously known (class of) solvable $N$-body models (41). There are however two other possibilities, which do yield two new classes of integrable systems.

The first possibility is to set $a_{11}=0$ (but $a_{12} \neq 0$ ) and to then require that the system of evolution equations (23) be consistent with the constraint (9). It is indeed plain that the system (23) becomes then linear:

$$
\begin{array}{r}
\ddot{c}_{m}-(N+1-m) a_{4} \dot{c}_{m-1}-\left[(N-m) a_{5}+a_{1}\right] \dot{c}_{m}+m a_{6} \dot{c}_{m+1} \\
+(N+1-m)(N+2-m) a_{7} c_{m-2}+(N+1-m)\left[(N-m) a_{8}+a_{2}\right] c_{m-1} \\
-m\left[(2 N-1-m) a_{9}+a_{3}\right] c_{m}+m(m+1) a_{10} c_{m+1} \\
=a_{12}(N+2-m) c_{m-2}
\end{array}
$$

and it is moreover easily seen (by setting $m=N-1$ ) that this system is consistent with the constraint (9), provided the parameters $a_{j}$ satisfy the following restrictions:

$$
a_{4}=a_{6}=2 a_{7}-a_{12}=a_{8}+a_{2}=a_{10}=a_{11}=0,
$$


entailing that the system (23) becomes

$$
\begin{array}{r}
\ddot{c}_{m}-\left[(N-m) a_{5}+a_{1}\right] \dot{c}_{m} \\
+(N-1-m)(N+2-m) a_{7} c_{m-2}-(N+1-m)(N-1-m) a_{2} c_{m-1} \\
-m\left[(2 N-1-m) a_{9}+a_{3}\right] c_{m}=0,
\end{array}
$$

and likewise the system (24) becomes

$$
\begin{aligned}
\ddot{z}_{n}= & a_{1} \dot{z}_{n}+a_{2}+a_{3} z_{n}-\frac{2 a_{7}}{z_{n}} \\
& +\sum_{\substack{m=1 \\
m \neq n}}^{N}\left\{( z _ { n } - z _ { m } ) ^ { - 1 } \left[2 \dot{z}_{n} \dot{z}_{m}+\left(\dot{z}_{n}+\dot{z}_{m}\right) a_{5} z_{n}\right.\right. \\
& \left.\left.+2\left(a_{7}-a_{2} z_{n}+a_{9} z_{n}^{2}\right)\right]\right\} .
\end{aligned}
$$

It is easily seen that these systems coincide with (7) and (6) after the following trivial relabeling of the parameters: $a_{j}=A_{j}$ for $j=1,2,3,5 ; a_{7}=A_{4}$, $a_{9}=A_{6}$. And it is moreover plain that the constraint (9) corresponds to the constraint (8), since the relation (2) among the zeros $z_{n}(t)$ and the coefficients $c_{m}(t)$ of the polynomial $\psi$ clearly entails 2

$$
c_{N}(t)=(-)^{N} \prod_{n=1}^{N} z_{n}(t), \quad c_{N-1}(t)=(-)^{N-1} \sum_{m=1}^{N} \prod_{\substack{n=1 \\ n \neq m}}^{N} z_{n}(t),
$$

hence

$$
c_{N-1}(t)=-c_{N}(t) \sum_{n=1}^{N} \frac{1}{z_{n}(t)} .
$$

The second possibility is to set $a_{12}=0$ (but $a_{11} \neq 0$ ) and to then require that the system of evolution equations (23) be consistent with the constraint (18). It is indeed plain that the system (23) becomes then linear:

$$
\begin{array}{r}
\ddot{c}_{m}-\left[(N+1-m) a_{4}-a_{11}\right] \dot{c}_{m-1}-\left[(N-m) a_{5}+a_{1}\right] \dot{c}_{m}+m a_{6} \dot{c}_{m+1} \\
+(N+1-m)(N+2-m) a_{7} c_{m-2}+(N+1-m)\left[(N-m) a_{8}+a_{2}\right] c_{m-1} \\
-m\left[(2 N-1-m) a_{9}+a_{3}\right] c_{m}+m(m+1) a_{10} c_{m+1}=0,
\end{array}
$$

\footnotetext{
${ }^{2}$ Actually to reach these conclusions one needs the additional condition $c_{N}(t) \neq 0$, namely (see (29a) $) z_{n}(t) \neq 0$; but the fact that such a condition must be imposed initially, and that it shall subsequently hold for all regular solutions of the Newtonian equations of motion (6), is implied by the presence of the term proportional to $A_{4}$ in the right-hand side of these equations of motion.
} 
and it is moreover easily seen (by setting $m=N$ ) that this system is consistent with the constraint (18), provided the parameters $a_{j}$ satisfy the following restrictions:

$$
a_{4}-a_{11}=a_{7}=a_{2}=(N-1) a_{9}+a_{3}=a_{12}=0,
$$

entailing that the system (23) becomes

$$
\begin{array}{r}
\ddot{c}_{m}-(N-m) a_{4} \dot{c}_{m-1}-\left[(N-m) a_{5}+a_{1}\right] \dot{c}_{m}+m a_{6} \dot{c}_{m+1} \\
+(N+1-m)(N-m) a_{8} c_{m-1} \\
-m(N-m) a_{9} c_{m}+m(m+1) a_{10} c_{m+1}=0
\end{array}
$$

and likewise the system (24) becomes

$$
\begin{aligned}
\ddot{z}_{n}= & a_{1} \dot{z}_{n}-(N-1) a_{9} z_{n}-2(N-1) a_{10} z_{n}^{2}+a_{4} \frac{\dot{z}_{n}}{z_{n}} \\
& +\sum_{\substack{m=1 \\
m \neq n}}^{N}\left\{( z _ { n } - z _ { m } ) ^ { - 1 } \left[2 \dot{z}_{n} \dot{z}_{m}+\left(\dot{z}_{n}+\dot{z}_{m}\right)\left(a_{4}+a_{5} z_{n}\right)\right.\right. \\
& \left.\left.+a_{6}\left(\dot{z}_{n} z_{m}+\dot{z}_{m} z_{n}\right) z_{n}+2\left(a_{8} z_{n}+a_{9} z_{n}^{2}+a_{10} z_{n}^{3}\right)\right]\right\} .
\end{aligned}
$$

It is easily seen that these systems coincide with (16) and (15) after the following trivial relabeling of the parameters: $a_{j}=B_{j}$ for $j=1,4,5,6$; $a_{8}=B_{7}, a_{9}=B_{2}, a_{10}=B_{3}$. And it is moreover plain 3 that the constraint (18) corresponds to the constraint (17), see (29a).

\section{Behaviors of the $N$-body models belonging to the first class}

In this section we discuss - in somewhat more detail than done in the introductory Section 1 - the behavior of the solutions of $N$-body models belonging to the first class, see ([6).

\footnotetext{
${ }^{3}$ Again with the additional condition $c_{N}(t) \neq 0$, which need not be highlighted since the presence of the term proportional to $B_{4}$ in the right-hand side of the Newtonian equations of motion (15) guarantees automatically its validity for all regular solutions of this system of ODEs.
} 
The general solution of the linear system of ODEs (7) with (9) is given by the formula

$$
c_{m}(t)=\sum_{\ell=1}^{N}\left[\gamma_{\ell}^{(+)} v_{m}^{(\ell)(+)} \exp \left(\lambda_{\ell}^{(+)} t\right)+\gamma_{\ell}^{(-)} v_{m}^{(\ell)(-)} \exp \left(\lambda_{\ell}^{(-)} t\right)\right]
$$

Here the $N$-vectors $\underline{v}^{(\ell)( \pm)}$ are the $2 N$ eigenvectors corresponding to the $2 N$ eigenvalues $\lambda_{\ell}^{( \pm)}$of the (generalized) eigenvalue equation

$$
\left(\lambda^{2} \underline{\mathbf{1}}+\lambda \underline{M}^{(1)}+\underline{M}^{(2)}\right) \underline{v}=0
$$

where the $N \times N$ matrices $\underline{M}^{(1)}$ and $\underline{M}^{(2)}$ are defined componentwise as follows (see (7)):

$$
\begin{gathered}
M_{m, m}^{(1)}=-\left[(N-m) A_{5}+A_{1}\right], \\
M_{m, m}^{(2)}=-m\left[(2 N-m-1) A_{6}+A_{3}\right], \\
M_{m, m-1}^{(2)}=-(N+1-m)(N-1-m) A_{2}, \\
M_{m, m-2}^{(2)}=(N-1-m)(N+2-m) A_{4},
\end{gathered}
$$

with all other elements vanishing. As for the $2 N$ constants $\gamma_{\ell}^{( \pm)}$, they are arbitrary except for the two requirements

$$
\begin{gathered}
\sum_{\ell=1}^{N}\left[\gamma_{\ell}^{(+)} v_{N-1}^{(\ell)(+)}+\gamma_{\ell}^{(-)} v_{N-1}^{(\ell)(-)}\right]=0, \\
\sum_{\ell=1}^{N}\left[\gamma_{\ell}^{(+)} v_{N-1}^{(\ell)(+)} \lambda_{\ell}^{(+)}+\gamma_{\ell}^{(-)} v_{N-1}^{(\ell)(-)} \lambda_{\ell}^{(-)}\right]=0,
\end{gathered}
$$

which clearly correspond to the constraints (11) via (34). Of course, in the context of the initial-value problem, the $2 N$ constants $\gamma_{\ell}^{( \pm)}$are determined by the $2 N$ initial data $c_{m}(0), \dot{c}_{m}(0)$ (including (11)) via the system of $2 N$ linear equations

$$
c_{m}(0)=\sum_{\ell=1}^{N}\left[\gamma_{\ell}^{(+)} v_{m}^{(\ell)(+)}+\gamma_{\ell}^{(-)} v_{m}^{(\ell)(-)}\right]
$$




$$
\dot{c}_{m}(0)=\sum_{\ell=1}^{N}\left[\gamma_{\ell}^{(+)} v_{m}^{(\ell)(+)} \lambda_{\ell}^{(+)}+\gamma_{\ell}^{(-)} v_{m}^{(\ell)(-)} \lambda_{\ell}^{(-)}\right] .
$$

And in the context of the initial-value problem for the Newtonian $N$-body model (6) the initial values $c_{m}(0), \dot{c}_{m}(0)$ are determined by the initial values $z_{n}(0), \dot{z}_{n}(0)$ via the following relations implied by (2):

$$
\begin{gathered}
\prod_{n=1}^{N}\left[z-z_{n}(0)\right]=z^{N}+\sum_{m=1}^{N} c_{m}(0) z^{N-m}, \\
-\sum_{n=1}^{N} \dot{z}_{n}(0) \prod_{\substack{m=1 \\
m \neq n}}^{N}\left[z-z_{m}(0)\right]=\sum_{m=1}^{N} \dot{c}_{m}(0) z^{N-m} .
\end{gathered}
$$

Remark: let us emphasize that, while in the context of the initial-value problem for the $N$-body problem (6) the constants $\gamma_{\ell}^{( \pm)}$depend on the initial data (as just explained), the $2 N$ eigenvalues $\lambda_{\ell}^{( \pm)}$- characterizing via (34) the time evolution of the coefficients $c_{m}(t)$ of the polynomial $\psi(z, t)$ whose $N$ zeros $z_{n}(t)$ yield the coordinates of the $N$ moving particles - do not depend on the initial data, but only on the parameters $A_{j}$ that specify the $N$-body model (6) under consideration.

We now notice that the $N \times N$ matrix $\underline{M}^{(1)}$ is diagonal, and the $N \times N$ matrix $\underline{M}^{(2)}$ is triangular $\left(M_{n m}^{(2)}=0\right.$ if $\left.n<m\right)$. Hence the eigenvalues $\lambda_{m}^{( \pm)}$ are just the roots of the $N$ (decoupled) second-order equations

$$
\lambda^{2}-\left[(N-m) A_{5}+A_{1}\right] \lambda-m\left[(2 N-m-1) A_{6}+A_{3}\right]=0,
$$

i. e.

$$
\begin{aligned}
\lambda_{m}^{( \pm)}=\frac{1}{2}\left\{(N-m) A_{5}+A_{1} \pm \Delta_{m}\right\} \\
\Delta_{m}^{2}=\left[(N-m) A_{5}+A_{1}\right]^{2}+4 m\left[(2 N-m-1) A_{6}+A_{3}\right] \\
=\left(N A_{5}+A_{1}\right)^{2}+\left(A_{5}^{2}-4 A_{6}\right) m^{2} \\
+2\left[2 A_{3}+2(2 N-1) A_{6}-A_{5}\left(A_{1}+N A_{5}\right)\right] m .
\end{aligned}
$$

The behavior of the solutions of the $N$-body problem (6) with (8) is given by the time evolution of the $N$ zeros $z_{n}(t)$ of the polynomial $\psi(z, t)$, see (2), whose coefficients evolve exponentially in time as entailed by (34) with (42). The study of the time evolution of the particle coordinates $z_{n}(t)$ is therefore 
reduced to the study of the motion of the zeros of a polynomial whose coefficients depend exponentially on time. In the generic case - characterized by exponents not all of which are purely imaginary - the asymptotic behavior of these zeros in the remote past and future - characterizing the qualitative behavior of the $N$-body model under consideration - can therefore be easily evinced by the treatment provided in Appendix G (entitled "Asymptotic behavior of the zeros of a polynomial whose coefficients diverge exponentially") of Ref. [2]. The subclass of $N$-body models (6) characterized by parameters $A_{j}$ satisfying the following restrictions,

$$
\begin{gathered}
A_{1}=\mathrm{i} \alpha, \quad A_{3}=\gamma, \quad A_{5}=\mathrm{i} \beta, \quad A_{6}=\eta \\
{[(N-m) \beta+\alpha]^{2}-4 m[(2 N-m-1) \eta+\gamma]>0, \quad m=1, \ldots, N,}
\end{gathered}
$$

(where the 4 constants $\alpha, \beta, \gamma, \eta$ are all real) - conditions which are necessary and sufficient to guarantee that all the $2 N$ eigenvalues $\lambda_{m}^{( \pm)}$are imaginary numbers - is instead clearly characterized by the property that all motions are confined. The more special subcase in which all the $2 N$ eigenvalues $\lambda_{m}^{( \pm)}$ are integer multiples of a single quantity $\mathrm{i} \omega$ with $\omega$ a positive constant, $\omega>0$, so that all the coefficients $c_{m}(t)$ are periodic with the same period $T=2 \pi / \omega$, is discussed in Section 5 .

\section{Behaviors of the $N$-body models belonging to the second class}

In this section we discuss - in somewhat more detail than done in the introductory Section 1; but rather tersely, to avoid repetitions of developments already elaborated in the preceding Section 3 - the behavior of the solutions of $N$-body models belonging to the second class, see (15).

The general solution of the linear system of ODEs (16) with (18) is given by a formula entirely analogous to (34), and the subsequent developments are also analogous to those reported in the preceding section, except that now the $N \times N$ matrices $\underline{M}^{(1)}$ and $\underline{M}^{(2)}$ are defined componentwise as follows (see (16)):

$$
\begin{gathered}
M_{m, m+1}^{(1)}=m B_{6}, \\
M_{m, m}^{(1)}=-\left[(N-m) B_{5}+B_{1}\right],
\end{gathered}
$$




$$
\begin{gathered}
M_{m, m-1}^{(1)}=-(N-m) B_{4}, \\
M_{m, m+1}^{(2)}=m(m+1) B_{3}, \\
M_{m, m}^{(2)}=-m(N-m) B_{2}, \\
M_{m, m-1}^{(2)}=(N+1-m)(N-m) B_{7},
\end{gathered}
$$

with all other elements vanishing. As for the $2 N$ constants $\gamma_{\ell}^{( \pm)}$, they are again arbitrary except now for the single requirement

$$
\sum_{\ell=1}^{N}\left[\gamma_{\ell}^{(+)} v_{N}^{(\ell)(+)} \lambda_{\ell}^{(+)}+\gamma_{\ell}^{(-)} v_{N}^{(\ell)(-)} \lambda_{\ell}^{(-)}\right]=0
$$

which clearly corresponds to the constraint (20) via (34).

Since the $N \times N$ matrices $\underline{M}^{(1)}$ and $\underline{M}^{(2)}$ are now neither diagonal nor triangular, the computations of the eigenvalues $\lambda_{m}^{( \pm)}$cannot be done now in explicit form (in the general case, with the coefficients $B_{j}$ appearing in (44) unrestricted). Therefore a discussion of the actual behavior of the solutions of this second solvable model cannot be done here in this general case to the same explicit extent as in the case of the first class of models, treated in the preceding section. There is however a subcase of this second model for which the treatment becomes closely analogous to that of the preceding section. Indeed clearly if

$$
B_{3}=B_{6}=0 \text {, }
$$

the two $N \times N$ matrices $\underline{M}^{(1)}$ and $\underline{M}^{(2)}$ become both triangular, hence the corresponding eigenvalue equation becomes again a second-order algebraic equation that can be easily solved 4 . Indeed it is easily seen that if the restriction (46) holds, this second-order equation determining the exponents $\lambda_{m}^{( \pm)}$that characterize the behavior of the second class of models coincides with (a subcase of) the second-order equation (41) determining the exponents $\lambda_{m}^{( \pm)}$that characterize the behavior of the first class of models, provided one sets

$$
A_{1}=B_{1}, \quad A_{5}=B_{5}, \quad A_{6}=B_{2}, \quad A_{3}+(N-1) A_{6}=0,
$$

\footnotetext{
${ }^{4}$ The $N \times N$ matrices $\underline{M}^{(1)}$ and $\underline{M}^{(2)}$ become triangular also in the case $B_{4}=B_{7}=0$, but whenever $B_{4}$ vanishes the novelty of the case treated in this paper disappears, so we do not pursue this case.
} 
hence the discussion given above for the first class of models becomes applicable to the second class of models provided they are restricted by the condition (46). And this includes of course also the more special, entirely isochronous, subcase, as discussed in Section 5.

\section{Isochronous versions of the solvable models}

A current definition of isochronous systems (see, for instance, [4]) attributes this property to any dynamical system that possesses at least one open (hence fully-dimensional) region in its phase space within which all solutions are completely periodic (i. e., periodic in all dependent variables) with the same fixed period (of course, the period being independent of the initial data, as long as they stay within that isochronicity region). The class of isochronous systems is vast [4], and it includes a large zoo of systems interpretable as $N$-body problems characterized by autonomous equations of motion of Newtonian type. A class of such isochronous systems is characterized by the Newtonian equations of motion (see Example 4.1.2-3 in Ref. [4])

$$
\underline{\ddot{z}}=-i \omega \underline{\dot{z}}+\sum_{k=1}^{K} \underline{F}^{(-k)}(\underline{z}, \underline{\dot{z}}+i \omega \underline{z}) \text {. }
$$

Here underlined variables indicate $N$-vectors, $\omega$ is a nonvanishing real constant, $K$ is an arbitrary positive integer, and the $K$ ( $N$-vector-valued) functions $\underline{F}^{(-k)}(\underline{z}, \underline{z})$ are required to be analytic (but not necessarily holomorphic) in all their $2 N$ arguments and to satisfy the scaling property

$$
\underline{F}^{(-k)}(c \underline{z}, \underline{\tilde{z}})=c^{-k} \underline{F}^{(-k)}(\underline{z}, \underline{\tilde{z}}), \quad k=1, \ldots, K .
$$

Indeed it has been shown [4] that these dynamical systems, (48), are isochronous, possessing an open (hence fully-dimensional) region in their phase space in which all their solutions are completely periodic with period

$$
T=\frac{2 \pi}{\omega} .
$$

It is easily seen that the system (24) belongs to this class, (48), with $K=1$, provided 


$$
\begin{aligned}
& a_{1}=-(2 N-1) \mathrm{i} \omega, \quad a_{2}=\left[a_{11}-(N-1) a_{4}\right] \mathrm{i} \omega, \\
& a_{3}=2(N-1) \omega^{2}, \quad a_{5}=2 \mathrm{i} \omega, \\
& a_{6}=0, \quad a_{8}=\mathrm{i} \omega a_{4}, \\
& a_{9}=-\omega^{2}, \quad a_{10}=0,
\end{aligned}
$$

while the constants $a_{4}, a_{7}, a_{11}$ and $a_{12}$ remain arbitrary. It is clear that both systems, (6) respectively (15), fall within this class, provided the constants $A_{j}$ respectively $B_{j}$ featured by these two solvable models satisfy appropriate restrictions (whose explicit determination can be left as a simple exercise for the diligent reader).

But here we like to use the more restrictive definition of entire isochronicity [4], stating that a dynamical system is entirely isochronous if all its nonsingular solutions are completely periodic with period $T$, or possibly with a (not arbitrarily large; indeed, generally rather small [12]) integer multiple of $T$-implying that all nonsingular solutions of an entirely isochronous system are in fact completely periodic with the same period, which however need not be the primitive period for all of them; or, equivalently, that the property of isochronicity holds in the entire phase space (with the possible exceptions of a lower-dimensional set of initial data yielding singular solutions).

One can then assert (see Appendix B for a proof) that the $N$-body problem (6) is indeed entirely isochronous provided the 6 constants $A_{j}$ it features satisfy the following 4 restrictions:

$$
\begin{aligned}
& A_{1}=\left(k_{1}-N k_{2}\right) \mathrm{i} \omega \\
& A_{3}=\frac{1}{2}\left(k_{2}+k_{3}\right)\left[\left(N-\frac{1}{2}\right)\left(k_{2}-k_{3}\right)-k_{1}\right] \omega^{2}, \\
& A_{5}=k_{2} \mathrm{i} \omega \\
& A_{6}=\frac{k_{3}^{2}-k_{2}^{2}}{4} \omega^{2},
\end{aligned}
$$

where $\omega$ is a positive constant, $\omega>0$ and the 3 numbers $k_{1}, k_{2}, k_{3}$ are integers, unrestricted (i. e., positive, negative or vanishing) except for the requirement

$$
k_{1} \neq-m k_{3} \text { for } m=1, \ldots, N \text {. }
$$

Note that the two constants $A_{2}$ and $A_{4}$ remain completely arbitrary. Also note that, if the two integers $k_{1}$ and $k_{2}$ vanish - entailing that $A_{1}$ and $A_{5}$ 
vanish, $A_{1}=A_{5}=0$, and $A_{3}=(1-2 N) A_{6}$, the only remaining restriction on $A_{6}$ being that it be a positive real number, $A_{6}>0$ - then the equations of motion (6) become real (of course provided real values are also assigned to the two a priori arbitrary constants $A_{2}$ and $A_{4}$ ).

But there is a second, different class of entirely isochronous systems, that is obtained from (a subclass of) the solvable system (6) with (8) via the standard "trick" procedure (see for instance Ref. [4]), as we now explain. To this end we take as starting point the following special case of the model (6) :

$$
\zeta_{n}^{\prime \prime}=-\frac{2 A_{4}}{\zeta_{n}}+2 \sum_{m=1, m \neq n}^{N} \frac{\zeta_{n}^{\prime} \zeta_{m}^{\prime}+A_{4}}{\zeta_{n}-\zeta_{m}}
$$

which corresponds to (6) with all the constants $A_{j}$ set to zero except $A_{4}$ (and moreover the purely notational replacement - convenient for what shall immediately follow - of the dependent variables $z_{n}(t)$ with the dependent variables $\zeta_{n}(\tau)$, and of the independent variable $t$ with the independent variable $\tau$, appended primes denoting of course differentiations with respect to this new independent variable $\tau$ ). We then set

$$
z_{n}(t)=\exp (-\mathrm{i} \omega t) \zeta_{n}(\tau), \quad \tau=\frac{\exp (\mathrm{i} \omega t)-1}{\mathrm{i} \omega},
$$

with $\omega$ a positive constant, and we thereby obtain the new $N$-body model characterized by the (autonomous) Newtonian equations of motion

$$
\begin{array}{r}
\ddot{z}_{n}=-\mathrm{i} \omega \dot{z}_{n}-\frac{2 A_{4}}{z_{n}} \\
+2 \sum_{\substack{m=1 \\
m \neq n}}^{N}\left\{\left(z_{n}-z_{m}\right)^{-1}\left[\left(\dot{z}_{n}+\mathrm{i} \omega z_{n}\right)\left(\dot{z}_{m}+\mathrm{i} \omega z_{m}\right)+A_{4}\right]\right\} .
\end{array}
$$

Due to the way this model has been obtained it is clear that it describes an entirely isochronous $N$-body problem - provided its initial data are constrained by the conditions (10), guaranteeing the validity of (8) (both for this model and for the model (52) ). It is also easy to verify that this model is not a special case of the entirely isochronous $N$-body problem identified above, i. e. (6) with (51) (and of course with (8) ).

Let us turn now to a discussion of entirely isochronous variants of the second class of solvable models treated in this paper. The identification of 
such models cannot be explicitly achieved in the general case along the same lines as done above - in the initial part of this section - for models belonging to the first class of solvable systems, because in this second case one cannot obtain explicitly the exponents $\lambda_{m}^{( \pm)}$, as explained in the preceding section (see the paragraph after (35) unless the restriction (46) holds, in which case the treatment given in the first part of this section for the first class of system can be immediately extended to the second class via the relations (47), which however entail (see the last of these equations (47)) that the restrictions (51) must now be complemented by the additional restriction

$$
k_{2}+k_{3}=0 \quad \text { or } \quad 2 k_{1}=N\left(k_{2}-k_{3}\right) .
$$

It is moreover again possible, also for the models of the second class, to proceed via the standard trick procedure. Since this approach is quite analogous to that described immediately above for the first class, we deal with it quite tersely.

The starting point is now the solvable system (15) with all constants vanishing except $B_{4}$, which we now write as follows:

$$
\zeta_{n}^{\prime \prime}=B_{4} \frac{\zeta_{n}^{\prime}}{\zeta_{n}}+\sum_{m=1, m \neq n}^{N} \frac{2 \zeta_{n}^{\prime} \zeta_{m}^{\prime}+B_{4}\left(\zeta_{n}^{\prime}+\zeta_{m}^{\prime}\right)}{\zeta_{n}-\zeta_{m}} .
$$

It is now again appropriate to use the version (53) of the trick, obtaining thereby the $N$-body problem - clearly entirely isochronous, because of the way it is obtained - characterized by the following equations of motion:

$$
\begin{array}{r}
\ddot{z}_{n}=-i \omega \dot{z}_{n}+B_{4} \frac{\dot{z}_{n}+i \omega z_{n}}{z_{n}} \\
+\sum_{\substack{m=1 \\
m \neq n}}^{N} \frac{2\left(\dot{z}_{n}+i \omega z_{n}\right)\left(\dot{z}_{m}+i \omega z_{m}\right)+B_{4}\left[\dot{z}_{n}+\dot{z}_{m}+i \omega\left(z_{n}+z_{m}\right)\right]}{z_{n}-z_{m}} .
\end{array}
$$

\section{Numerical results}

In this Section we perform a numerical integration of the equations of motion (6) of the first model in order to illustrate the previous findings. The numerical integration has been done with an embedded Runge-Kutta method of 
order 8(5,3) with automatic step size control, as developed by Prince and Dormand [13]. The integration and the graphical output have been performed with the software Dynamics solver ${ }^{5}$ developed by J. Aguirregabiria.

In order to simplify our numerical treatment we have set in (6) $N=3$, and $A_{1}=A_{2}=A_{5}=0$. Hence, our numerical study focuses on the following system of three nonlinear complex ODEs of second order:

$$
\ddot{z}_{n}=A_{3} z_{n}-\frac{2 A_{4}}{z_{n}}+\sum_{\substack{m=1 \\ m \neq n}}^{3}\left(z_{n}-z_{m}\right)^{-1}\left[2 \dot{z}_{n} \dot{z}_{m}+2\left(A_{4}+A_{6} z_{n}^{2}\right)\right] .
$$

\section{Generic (chaotic) behaviour}

When $A_{4}=0$ this system is solvable and it is a particular case of the family discussed in Chapter 2.3.3 of [2]. When $A_{4} \neq 0$ and no restrictions are imposed on the initial data, the system (58) corresponds to the motion of the zeros of a $3^{\text {rd }}$ degree polynomial whose coefficients evolve in time according to the nonlinear system (23). The system behaves in general in a chaotic manner as it can be evinced from the numerical results in Figures 1e, $\mathbf{1 f}$ and 2. The initial data for that integration have been chosen to be

$$
\begin{array}{ll}
z_{1}(0)=1.5-2 \mathrm{i}, & \dot{z}_{1}(0)=2-\mathrm{i} \\
z_{2}(0)=-1+0 \mathrm{i}, & \dot{z}_{2}(0)=-1+\mathrm{i} \\
z_{3}(0)=1.2+0.4 \mathrm{i}, & \dot{z}_{3}(0)=2.32-0.76 \mathrm{i}
\end{array}
$$

which do not satisfy the constraints (10), while the values of the coefficients are

$$
A_{3}=-5.1 \pi^{2}, \quad A_{4}=15, \quad A_{6}=\pi^{2} .
$$

\section{Quasi-periodic behaviour}

However, for the same values of these parameters, (60), initial data can be chosen within the algebraic submanifold defined by (10). One such assignment is

$$
\begin{array}{ll}
z_{1}(0)=2-2 \mathrm{i}, & \dot{z}_{1}(0)=2-\mathrm{i} \\
z_{2}(0)=-1+0 \mathrm{i}, & \dot{z}_{2}(0)=-1+\mathrm{i}, \\
z_{3}(0)=1.2+0.4 \mathrm{i}, & \dot{z}_{3}(0)=2.32-0.76 \mathrm{i} .
\end{array}
$$

\footnotetext{
${ }^{5}$ This program is available at http://tp.lc.ehu.es/jma/ds/ds.html
} 
As discussed in Section 2, now the system (58) is solvable, and it corresponds to the motion of the zeros of a monic $3^{\text {rd }}$ degree polynomial whose coefficients evolve in time according to the following linear system:

$$
\begin{aligned}
\ddot{c}_{1}-\left(4 A_{6}+A_{3}\right) c_{1} & =0, \\
c_{2} & =0, \\
\ddot{c}_{3}-2 A_{4} c_{1}-3\left(2 A_{6}+A_{3}\right) c_{3} & =0 .
\end{aligned}
$$

The solution of this system is trivial and from (43) and the discussion in Section 4 , we see that it suffices that

$$
4 A_{6}+A_{3}<0, \quad 2 A_{6}+A_{3}<0
$$

for the orbits to be confined. As it follows from (34) with (42), the coefficients of the polynomial are in general quasi-periodic functions of time, hence also the behaviour of the system (10) is quasi-periodic as it can be seen in Figures 1c, $\mathbf{1 d}$ and $\mathbf{2}$, where we have again assigned the values (60) of the parameters, and the same initial data (61).

\section{Isochronous behaviour}

Following the discussion of Section 5 and Appendix B, the family of solvable problems discussed above includes a subclass of entirely isochronous systems. They occur when all the frequencies of the system are commensurable. A look at (51) reveals that these conditions are met if we choose

$$
A_{1}=A_{5}=0, \quad A_{3}=-\frac{5}{4} k_{3}^{2} \omega^{2}, \quad A_{6}=\frac{1}{4} k_{3}^{2} \omega^{2} .
$$

We have set $k_{3}=1$ and $\omega=2 \pi$ so that the fundamental period is $T=1$. The choice of parameters is then

$$
A_{3}=-5 \pi^{2}, \quad A_{4}=15, \quad A_{6}=\pi^{2} .
$$

and the initial data are the same as in the previous case (61). The resulting motions are shown in Figures $\mathbf{1 a}, \mathbf{1 b}$ and $\mathbf{2}$.

\section{Outlook}

The novel cases treated in this paper do not exhaust all the possible solvable many-body problems associated to the motion of the roots of polynomials 
whose coefficients satisfy a linear system of ODEs. This question is related to the classification of exceptional subspaces $X_{k}$ with co-dimension $k>1$, which has not yet been completed. However, as mentioned in Appendix A, some results based on $X_{2}$ spaces and operators will be reported soon [5].

\section{Acknowledgements}

The results reported in this paper where obtained during a visit in October 2006 of one of us (DGU) to the Department of Physics of the University of Rome "La Sapienza", performed in the context of the Collaboration Agreement among the University of Rome "La Sapienza" and the Universidad Complutense of Madrid. The research of DGU is supported in part by the Ramón y Cajal program of the Ministerio de Ciencia y Tecnología and by the DGI under grants FIS2005-00752 and MTM2006-00478. It is a pleasure to acknowledge illuminating discussions with Robert Milson.

\section{Appendix A}

In this Appendix we explain the general method to write down certain PDEs in $1+1$ dimensions possessing solutions which are monic polynomials $\psi(z, t)$ of degree $n$ in $z$ whose $N$ coefficients are time-dependent functions $c_{m}(t)$. The requirement to ensure the solvability of the nonlinear $N$-body problem characterizing the time evolution of the $N$ zeros $z_{n}(t)$ of such a polynomial is that the coefficients $c_{m}(t)$ evolve according to a linear system of ODEs.

Let us denote by $\mathcal{P}_{N}$ the vector space of polynomials in $z$ of degree less than or equal to $N$ :

$$
\mathcal{P}_{N}=\operatorname{span}\left\{1, z, z^{2}, \ldots, z^{N}\right\}, \quad \operatorname{dim} \mathcal{P}_{N}=N+1 .
$$

We shall denote also by $\mathcal{D}_{2}\left(\mathcal{P}_{N}\right)$ the vector space of differential operators of order 2 in $z$ with analytic coefficients that leave the space $\mathcal{P}_{N}$ invariant, i.e.

$$
\mathcal{D}_{2}\left(\mathcal{P}_{N}\right)=\left\{T=a_{2}(z) D_{z z}+a_{1}(z) D_{z}+a_{0}(z) \mid T \mathcal{P}_{N} \subset \mathcal{P}_{N}\right\}
$$

Notation: here and below we use the notation $D_{z} \equiv \partial / \partial z, D_{z z} \equiv \partial^{2} / \partial z^{2}$, $D_{t} \equiv \partial / \partial t, D_{t t} \equiv \partial^{2} / \partial t^{2}, D_{z t} \equiv \partial^{2} / \partial z \partial t$. 
Consider the action of the most general linear second-order partial differential operator in $z$ and $t$ :

$$
L=a_{1} D_{t t}+a_{2} D_{z t}+a_{3} D_{z z}+a_{4} D_{z}+a_{5} D_{t}+a_{6}, \quad a_{i}=a_{i}(z, t)
$$

on the following time-dependent polynomial of degree $N$ in $z$ (see (2) ),

$$
\psi(z, t)=\prod_{n=1}^{N}\left[z-z_{n}(t)\right]=z^{N}+\sum_{m=1}^{N} c_{m}(t) z^{N-m} .
$$

The restriction to second-order operators ensures that the nonlinear manybody problem for the zeros will have at most two-body interactions, which is the more physically interesting case. If many-body interactions are allowed, it is obvious that the class of such solvable systems that can be constructed with this method is larger. Since we want the evolution of the coefficients $c_{m}(t)$ to be a linear autonomous (hence solvable) system, we assume $a_{i}(z, t)=a_{i}(z)$ and without loss of generality we also set $a_{1}(z)=1$.

We now examine the conditions on $a_{i}(z)$ such that the equation $L[\psi]=0$ implies a linear system for $c_{m}(t)$. These conditions arise from imposing that $L$ acting on $\psi(z, t)$ produces a polynomial of degree $N-1$. They are satisfied by a second-order differential operator in $z$ and $t$ only in the following cases:

- the second-order differential operator $D_{t t}$;

- one of the following 4 differential operators of first-order in $t$ :

$$
D_{t}, \quad D_{z t}, \quad z D_{z t}, \quad z\left[z D_{z t}-(N-1) D_{t}\right] ;
$$

- a second-order differential operator in $z$ that maps $\mathcal{P}_{N}$ to $\mathcal{P}_{N-1}$.

The characterization of vector spaces of linear (and nonlinear) differential operators in one and several variables of any given order that map $\mathcal{P}_{N}$ to $\mathcal{P}_{N-k}$ has been treated in [9]. Working out all the possible cases for a second order differential operator in one variable that maps $\mathcal{P}_{N}$ to $\mathcal{P}_{N-1}$ is a simple exercise that produces the following result:

$$
\begin{aligned}
& T_{2}^{+1}=z\left(z D_{z}-N+1\right)\left(z D_{z}-N+2\right) \\
& T_{2}^{0}=z^{2} D_{z z}-N(N-1) \\
& T_{2}^{-1}=z D_{z z} \\
& T_{2}^{-2}=D_{z z} \\
& T_{1}^{0}=z D_{z}-N \\
& T_{1}^{-1}=D_{z}
\end{aligned}
$$


A linear combination of the eleven operators written above is precisely equation (11) of this paper, which was treated in Section 2.3.3 of [2] (and several specific cases were then investigated in Section 2.3.4). However, the question arises whether this is the most general class of PDEs in $z$ and $t$ which admit as solutions polynomials in $z$ whose coefficients are functions of $t$ that evolve according to a linear system. The recent discovery of the so-called exceptional polynomial subspaces of $\mathcal{P}_{N}$ shows that other PDEs with those properties exist (and therefore other solvable many-body problems) provided some constraints are imposed.

An exceptional polynomial subspace $\mathcal{M}_{N}^{(k)}$ of co-dimension $k$ in $\mathcal{P}_{N}$ is defined by the property that some second-order differential operators that preserve $\mathcal{M}_{N}^{(k)}$ do not preserve $\mathcal{P}_{N}$. More specifically, consider a space $\mathcal{M}_{N}^{(k)} \subset$ $\mathcal{P}_{N}$ generated by $N+1-k$ linearly independent polynomials, all of them of degree at most $N$ :

$$
\mathcal{M}_{N}^{(k)}=\operatorname{span}\left\{p_{1}(z), \ldots, p_{N+1-k}(z)\right\}
$$

We will say that $\mathcal{M}_{N}^{(k)}$ is an exceptional polynomial subspace of co-dimension $k$ in $\mathcal{P}_{N}$ (for short, an $X_{k}$-space) if $\mathcal{D}_{2}\left(\mathcal{M}_{N}^{(k)}\right) \not \subset \mathcal{D}_{2}\left(\mathcal{P}_{N}\right)$. Such exceptional subspaces do exist, and they provide novel differential operators with polynomial eigenfunctions. Exceptional subspaces have been first analyzed in the context of quasi-exactly solvable potentials in quantum mechanics $[7,10]$; they are also connected with the Darboux transformation [8] and with non classical families of orthogonal polynomials [11]. $X_{1}$-spaces have been fully classified arriving at the result that there is essentially one such subspace up to projective transformations. This space is precisely

$$
X_{1}=\operatorname{span}\left\{1, z^{2}, z^{3}, \ldots, z^{N}\right\},
$$

which can also be characterized as

$$
X_{1}=\left\{p \in \mathcal{P}_{N} \mid p^{\prime}(0)=0\right\} .
$$

It can be shown that $\operatorname{dim} \mathcal{D}_{2}\left(X_{1}\right)=7$. The most interesting element of this space is the operator

$$
D_{z z}-\frac{2}{z} D_{z}
$$

which preserves $X_{1}$ but not $\mathcal{P}_{N}$. The first class of models treated in this paper - see (6) - is related to this exceptional space $X_{1}$, and the novel term 
proportional to the constant $A_{4}$ in the many-body problem (6) is precisely that due to the inclusion of this operator. The constraint on the many-body problem (8) is precisely the one that defines the exceptional subspace $X_{1}$. The second class of many-body models treated above has a different origin, not related to the exceptional subspaces of $\mathcal{P}_{N}$, being instead associated with the role of the time-derivative, see (69).

Exceptional subspaces of higher co-dimension exist but a full classification is not yet available. Some new many-body problems with constraints associated to $X_{2}$-spaces will be treated in a forthcoming publication [5], their main novelty being that the coefficients of the new differential operators are not only inverse powers but rational functions of $z$.

\section{Appendix B}

In this Appendix we justify the assertion made in Section 5, that the conditions (51) guarantee that the $N$-body model (6) with (10) is entirely isochronous.

Indeed, clearly this $N$-body problem is entirely isochronous if all the solutions of the corresponding linear problem (7) are completely periodic with the same period $T$ (readers for whom this is not clear are advised to consult, say, Ref. [4]). Clearly a necessary and sufficient condition for this to happen is that the $2 N$ eigenvalues $\lambda_{m}^{( \pm)}$, see (42), be all integer multiples of a common imaginary constant, say

$$
\lambda_{m}^{( \pm)}=k_{m}^{( \pm)} \mathrm{i} \omega
$$

with $\omega$ a positive constant, $\omega=2 \pi / T>0$, and the coefficients $k_{m}^{( \pm)}$all integers, and moreover that

$$
\lambda_{m}^{(+)} \neq \lambda_{m}^{(-)}
$$

for all values of the index $m$, i. e. for $m=1, \ldots, N$. Clearly the first of these two relations, (74a), can only be true if the quantity $\Delta_{m}^{2}$, see (42b), is a perfect square for all values of $m$, so that

$$
\Delta_{m}=\alpha+\beta m
$$

clearly entailing (see (42b))

$$
\alpha=N A_{5}+A_{1}, \quad \beta^{2}=A_{5}^{2}-4 A_{6},
$$


and (as we hereafter assume)

$$
2 A_{3}+2(2 N-1) A_{6}-A_{5}\left(N A_{5}+A_{1}\right)=\alpha \beta .
$$

Via (42) these formulas yield

$$
\lambda_{m}^{(+)}=\alpha+\frac{m}{2}\left(\beta-A_{5}\right), \quad \lambda_{m}^{(-)}=-\frac{m}{2}\left(\beta+A_{5}\right) .
$$

Hence (74a) entails

$$
\alpha=k_{1} \mathrm{i} \omega, \quad \beta-A_{5}=2 j \mathrm{i} \omega, \quad \beta+A_{5}=2 k \mathrm{i} \omega,
$$

with $k_{1}, j, k$ integers, and the (sum and difference of the) last two formulas entail

$$
A_{5}=k_{2} \mathrm{i} \omega, \quad \beta=k_{3} \mathrm{i} \omega,
$$

with $k_{2}$ and $k_{3}$ integers. Hence the two equations (76a) yield

$$
A_{1}=\left(k_{1}-N k_{2}\right) \mathrm{i} \omega, \quad A_{6}=\frac{k_{3}^{2}-k_{2}^{2}}{4} \omega^{2},
$$

and from (76b) one finally gets

$$
A_{3}=\frac{1}{2}\left(k_{2}+k_{3}\right)\left[\left(N-\frac{1}{2}\right)\left(k_{2}-k_{3}\right)-k_{1}\right] \omega^{2} .
$$

The 4 formulas (51) are thereby proven. And it is moreover clear that the condition (74b) entails the requirement (51e), thereby completing the proof of the results reported in Section 5 . 


\section{References}

[1] F. Calogero, "Motion of poles and zeros of special solutions of nonlinear and linear partial differential equations, and Related "Solvable" Many Body Problems", Nuovo Cimento 43B, 177-241 (1978).

[2] F. Calogero, Classical many-body problems amenable to exact treatments, Lecture Notes in Physics Monograph m 66, Springer, 2001.

[3] F. Calogero, The "neatest" many-body problem amenable to exact treatments (a "goldfish"?), Physica D 152-153, 78-84 (2001).

[4] F. Calogero, Isochronous systems, 200-page monograph, to be published, 2007.

[5] F. Calogero and D. Gómez-Ullate, "Additional classes of solvable manybody problems of goldfish type with constraints", in preparation.

[6] D. Gómez-Ullate, N. Kamran, R. Milson, "The Darboux transformation and algebraic deformations of shape-invariant potentials", J. Phys. A 37, 1789-1804 (2004).

[7] D. Gómez-Ullate, N. Kamran, R. Milson, "Quasi-exact solvability and the direct approach to invariant subspaces", J. Phys. A 38, 2005-2019 (2005).

[8] D. Gómez-Ullate, N. Kamran, R. Milson, "Supersymmetry and algebraic Darboux transformations", J. Phys. A 37, 10065-10078 (2004).

[9] D. Gómez-Ullate, N. Kamran, R. Milson, "Structure theorems for linear and non-linear differential operators admitting invariant polynomial subspaces, Discrete and Cont. Din. Syst., in press, arXiV:nlin.SI/0604070.

[10] D. Gómez-Ullate, N. Kamran, and R. Milson, Quasi-exact solvability beyond the SL(2) algebraization, Phys. Atom. Nuclei, in press arXiv:nlin.SI/0601053.

[11] D. Gómez-Ullate, N. Kamran, and R. Milson, "A generalized Bochner problem and related orthogonal polynomials", in preparation. 
[12] D. Gómez-Ullate and M. Sommacal, "Periods of the goldfish many-body problem", J. Nonlinear Math. Phys. 12, Suppl. 1, 351-362 (2005).

[13] E. Hairer, S. P. Norsett and G. Wanner, Solving Ordinary Differential Equations I: Nonstiff Problems, Springer, Berlin (1987), p. 193-195. 

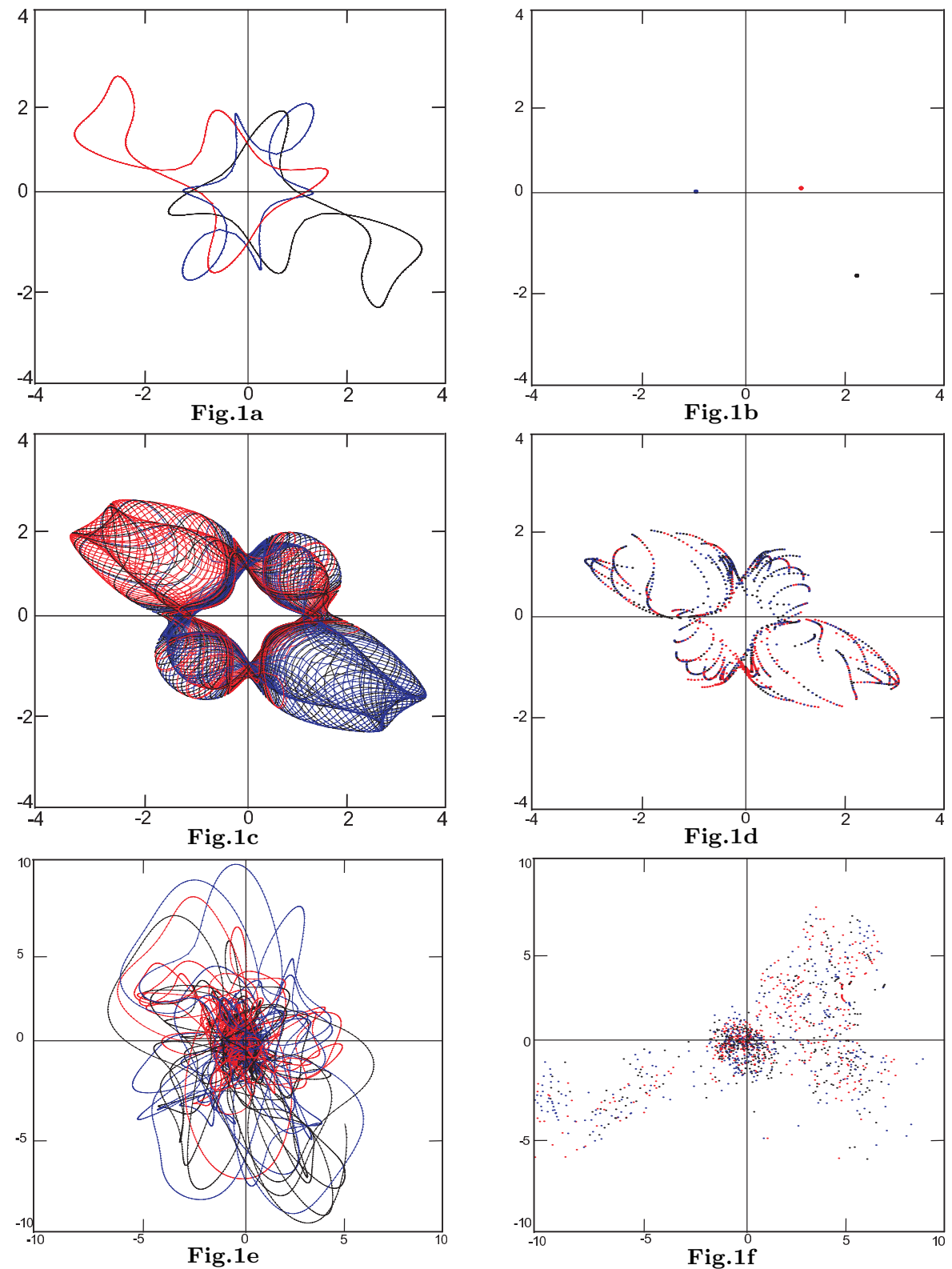

Figure 1: Left column: trajectories of the 3-body system (58) in the periodic (1a), quasi-periodic (1c) or chaotic regimes (1e). Right column: Stereographic projection of the trajectories: the positions of the three particles are plotted at every integer multiple of $T=1$ up to $10^{3}$ iterations. 

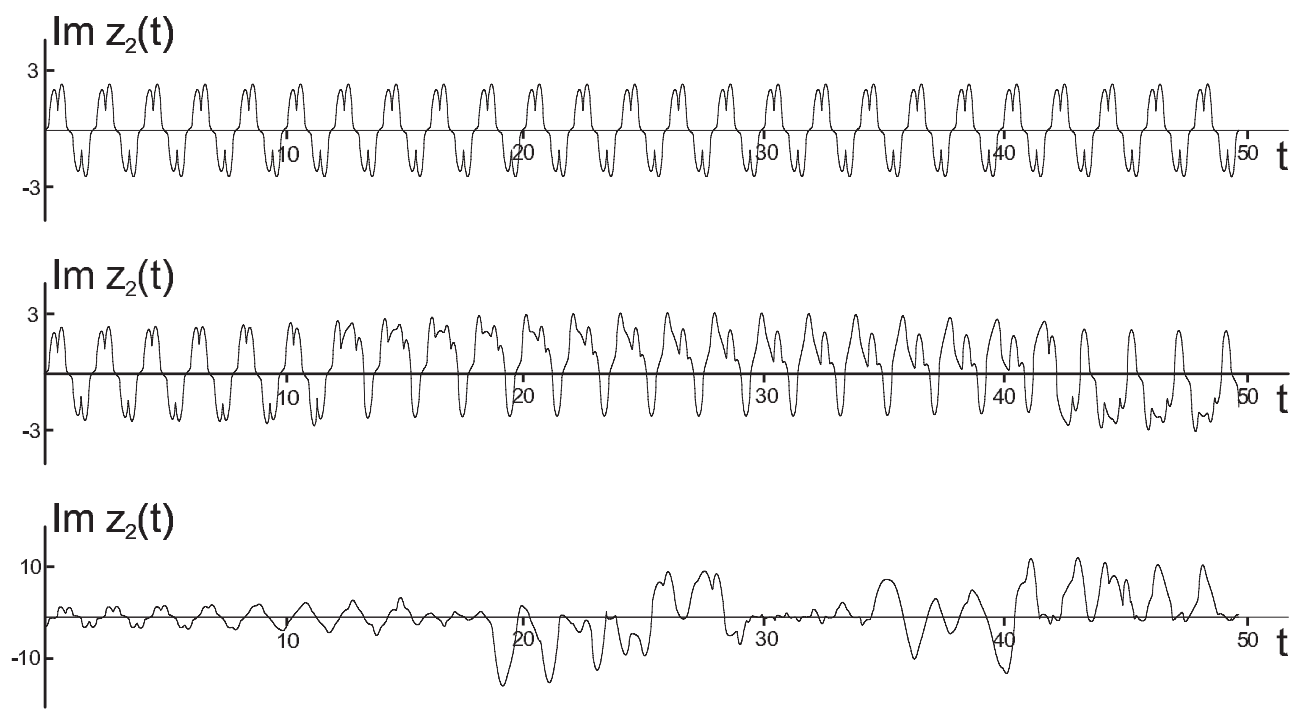

Figure 2: A plot of $\operatorname{Im} z_{2}(t)$ for the choice of parameters and initial data described in Section 6. The periodic, quasi-periodic and chaotic behaviours are manifest. 